PROCEEDINGS OF THE

AMERICAN MATHEMATICAL SOCIETY

Volume 132, Number 12, Pages 3503-3507

S 0002-9939(04)07517-3

Article electronically published on July 26, 2004

\title{
CONSECUTIVE CANCELLATIONS IN BETTI NUMBERS
}

\author{
IRENA PEEVA
}

(Communicated by Michael Stillman)

\begin{abstract}
Let $I$ be a homogeneous ideal in a polynomial ring over a field. By Macaulay's Theorem, there exists a lexicographic ideal $L$ with the same Hilbert function as $I$. We prove that the graded Betti numbers of $I$ are obtained from those of $L$ by a sequence of consecutive cancellations.
\end{abstract}

Throughout the paper, $S=k\left[x_{1}, \ldots, x_{n}\right]$ is a polynomial ring over a field $k$, and $I$ is a homogeneous ideal in $S$.

First, we recall the definition of a lexicographic ideal. A monomial ideal $M$ is called lexicographic if for every $j \in \mathbf{N}$ the space $M_{j}$ is spanned by the first $\operatorname{dim}\left(M_{j}\right)$ monomials in the lexicographic order. By Macaulay's Theorem [Ma], there exists a lexicographic ideal $L$ with the same Hilbert function as $I$. Throughout the paper we denote this ideal by $L$. It was proved by Bigatti, Hulett, and Pardue that the graded Betti numbers $\beta_{i, j}(S / L)$ are greater than or equal to the corresponding graded Betti numbers $\beta_{i, j}(S / I)$ (all graded Betti numbers are taken over $S$ ).

The Hilbert function can be computed from the graded Betti numbers as follows (cf. [Ei]):

$$
\begin{aligned}
& \sum_{j=0}^{\infty} \operatorname{dim}_{k}(S / I)_{j} t^{j}=\frac{\sum_{j=0}^{\infty} \sum_{i=0}^{n}(-1)^{i} \beta_{i, j}(S / I) t^{j}}{(1-t)^{n}} \\
& \sum_{j=0}^{\infty} \operatorname{dim}_{k}(S / L)_{j} t^{j}=\frac{\sum_{j=0}^{\infty} \sum_{i=0}^{n}(-1)^{i} \beta_{i, j}(S / L) t^{j}}{(1-t)^{n}} .
\end{aligned}
$$

These equalities imply that the graded Betti numbers $\beta_{i, j}(S / I)$ and $\beta_{i, j}(S / L)$ are related, as described below.

Given a sequence of numbers $\left\{c_{i, j}\right\}$, we obtain a new sequence by a cancellation as follows: fix a $j$, and choose $i$ and $i^{\prime}$ so that one of the numbers is odd and the other is even; then replace $c_{i, j}$ by $c_{i, j}-1$, and replace $c_{i^{\prime}, j}$ by $c_{i^{\prime}, j}-1$. We have a consecutive cancellation when $i^{\prime}=i+1$. If we need to be specific, we call it a consecutive $(i, j)$-cancellation. The term "consecutive" is justified by the fact that we consider cancellations in Betti numbers of consecutive homological degrees.

Received by the editors November 21, 2002 and, in revised form, August 25, 2003.

2000 Mathematics Subject Classification. Primary 13D02.

Key words and phrases. Syzygies. 
The equalities above imply that the graded Betti numbers $\beta_{i, j}(S / I)$ are related to the graded Betti numbers $\beta_{i, j}(S / L)$ by a sequence of cancellations. This was observed and applied by Evans-Richert [ER, Geramita-Harima-Shin (several papers), and Richert [Ri] in order to study the possible Betti diagrams of ideals with a fixed Hilbert function. We provide a more precise result:

Theorem 1.1. Let $I$ be a graded ideal and $L$ be the lexicographic ideal with the same Hilbert function. The graded Betti numbers $\beta_{i, j}(S / I)$ can be obtained from the graded Betti numbers $\beta_{i, j}(S / L)$ by a sequence of consecutive cancellations.

Proof. We can assume that the field $k$ is infinite, since extending the ground field is a faithfully flat functor.

Hartshorne [Ha] proved that the Hilbert scheme parametrizing all subschemes of $\mathbf{P}^{n-1}$ with a fixed Hilbert polynomial is connected. Modifications of his proof were introduced and used by Pardue and Reeves. We will use Pardue's proof $\mathrm{Pa}$ of the fact that $I$ and $L$ are connected by a sequence of deformations of the following three types:

(1) generic change of coordinates;

(2) deformation between an ideal and an initial ideal;

(3) polarization and then factoring out generic hyperplane sections; more precisely, applying $\sigma_{\mathbf{L}}^{\prime}$ defined in [Pa, Section 4].

Clearly, the graded Betti numbers are preserved under (1). It is well known, cf. [Pa, Corollary 15], that (3) preserves the graded Betti numbers as well.

It remains to consider (2). Let $T$ be a homogeneous ideal and $T^{\prime}$ be an initial ideal. By $[\mathrm{Ba}]$, we can choose a vector $\mathbf{w}=\left(w_{1}, \ldots, w_{n}\right)$ with strictly positive integer coordinates, such that $T^{\prime}$ is the initial ideal of $T$ with respect to the weight order induced by the weight vector w; cf. Ei Theorem 15.16]. Let $\tilde{T}$ be the homogenization of $T$ in the polynomial $\operatorname{ring} \tilde{S}=S[t]$; here $\tilde{S}$ is graded by $\operatorname{deg}\left(x_{i}\right)=$ $w_{i}$ for $1 \leq i \leq n$ and $\operatorname{deg}(t)=1$. Then $t$ and $t-1$ are regular elements on $\tilde{S} / \tilde{T}$; cf. [Ei, Theorem 15.17]. Denote by $\tilde{\mathbf{F}}$ a graded minimal free resolution of $\tilde{S} / \tilde{T}$ over $\tilde{S}$. Then $\tilde{\mathbf{F}} \otimes \tilde{S} / t$ is a minimal free resolution of $S / T^{\prime}=\tilde{S} / \tilde{T} \otimes \tilde{S} / t$. Thus, the graded Betti numbers of $S / T^{\prime}$ and $\tilde{S} / \tilde{T}$ coincide. On the other hand, $\tilde{\mathbf{F}} \otimes \tilde{S} /(t-1)$ is a non-minimal graded free resolution of $S / T=\tilde{S} / \tilde{T} \otimes \tilde{S} /(t-1)$. Therefore,

$$
\tilde{\mathbf{F}} \otimes \tilde{S} /(t-1) \cong \mathbf{F} \oplus \mathbf{G},
$$

where $\mathbf{F}$ is a minimal graded free resolution of $S / T$ and $\mathbf{G}$ is a trivial complex; cf. [Ei, Theorem 20.2]. The triviality of the complex $\mathbf{G}$ implies that the graded Betti numbers of $S / T$ are obtained from those of $\tilde{S} / \tilde{T}$ by consecutive cancellations.

Remark. Consider a flat family over $k[t]$ of quotients of $S$ whose fiber over 0 is $S / J$ and whose fiber over $\alpha \in k \backslash 0$ is $S / J^{\prime}$. The argument in the proof of Theorem 1.1 shows that the graded Betti numbers $\beta_{i, j}\left(S / J^{\prime}\right)$ can be obtained from the graded Betti numbers $\beta_{i, j}(S / J)$ by a sequence of consecutive cancellations. We also remark that Theorem 1.1 can be generalized and holds for modules.

It should be noted that there are many examples where the existence of possible consecutive cancellations does not imply the existence of an ideal for which those cancellations are realized.

In order to apply the theorem, we study when a consecutive cancellation in $\beta_{i, j}(S / L)$ is possible. For a monomial $m$, we set $\max (m)=\max \left\{p \mid x_{p}\right.$ divides $\left.m\right\}$. 
Proposition 1.2. Let $L$ be a lexicographic ideal. If a consecutive $(i, j)$-cancellation in the sequence $\beta_{i, j}(S / L)$ is possible, then the following two conditions are satisfied:

(1) $L$ has a minimal monomial generator $m$ of degree $j-i+1$ with $\max (m) \geq i$.

(2) $L$ has a minimal monomial generator $m^{\prime}$ of degree $j-i$ with $\max \left(m^{\prime}\right) \geq i+1$.

Proof. The minimal free graded resolution of $S / L$ is provided by a construction of Eliahou and Kervaire [EK]. The resolution has basis

$$
\left\{\left(m ; t_{1}, \ldots, t_{i}\right) \mid m \in G(L), 1 \leq t_{1}<\cdots<t_{i}<\max (m) \text { natural numbers }\right\},
$$

where $G(L)$ denotes the set of the canonical minimal monomial generators of $L$. The element $\left(m ; t_{1}, \ldots, t_{i}\right)$ has homological degree $i+1$ and an internal degree $i+\operatorname{deg}(m)$.

Thus, the first condition is equivalent to $\beta_{i, j}(S / L) \neq 0$, and the second condition is equivalent to $\beta_{i+1, j}(S / L) \neq 0$.

Corollaries 1.4 and 1.5 below are two immediate applications of Theorem 1.1.

The next corollary is on classes of Hilbert functions, such that all ideals with a fixed such Hilbert function have the same graded Betti numbers. An example of this type was discovered by Richert [Ri, Example 3.1]; his example is just a concrete ideal $L$ satisfying Corollary 1.4. Other examples were obtained by Geramita, Harima, and Shin GHS1, GHS2.

Corollary 1.4. Let $L$ be a lexicographic ideal. Suppose that $L$ does not have two minimal monomial generators in consecutive degrees. If $J$ is a homogeneous ideal with the same Hilbert function as $L$, then $J$ has the same graded Betti numbers as $L$.

Proof. By Proposition 1.2, it follows that no consecutive cancellation can be performed on the sequence of the graded Betti numbers of $S / L$. Apply Theorem 1.1.

For some classes of ideals, we provide a lower bound on the total Betti numbers in the spirit of the Buchsbaum-Eisenbud-Horrocks Conjecture.

Corollary 1.5. Let $L$ be a lexicographic ideal. Suppose that $L$ has a minimal monomial generator $m$ with $\max (m)=n$ and such that $L$ has no minimal monomial generators in degrees $\operatorname{deg}(m)-1$ and $\operatorname{deg}(m)+1$. Then

$$
\beta_{p}(S / J) \geq\left(\begin{array}{l}
n-1 \\
p-1
\end{array}\right) \quad \text { for } p \geq 1,
$$

for every homogeneous ideal $J$ with the same Hilbert function as $L$.

Proof. Since $L$ has no minimal monomial generators in degrees $\operatorname{deg}(m)-1$ and $\operatorname{deg}(m)+1$, it follows from Proposition 1.2 that, for each $i$, no consecutive cancellation can involve $\beta_{i, i+\operatorname{deg}(m)-1}(S / L)$. By Theorem 1.1, it follows that

$$
\beta_{i}(S / J) \geq \beta_{i, i+\operatorname{deg}(m)-1}(S / J)=\beta_{i, i+\operatorname{deg}(m)-1}(S / L) .
$$

Furthermore, since $L$ has a minimal monomial generator $m$ with $\max (m)=n$, we conclude that for each $i$ we have the inequality $\beta_{i, i+\operatorname{deg}(m)-1}(S / L) \geq\left(\begin{array}{c}n-1 \\ i-1\end{array}\right)$ by (1.3). 
Example 1.6. Take

$$
\begin{aligned}
& L=\left(x_{1}^{2}, x_{1} x_{2}, x_{1} x_{3}^{3}, x_{1} x_{3}^{2} x_{4}, x_{1} x_{3}^{2} x_{5},\right. \\
& \quad \text { any monomial generators of degree } \geq 6 \text { so that } L \text { is lexicographic }) .
\end{aligned}
$$

The monomial $m=x_{1} x_{3}^{2} x_{5}$ satisfies the condition in Corollary 1.4. Therefore, for every homogeneous ideal $J$ with the same Hilbert function as $L$ we have that $\beta_{p}(S / J) \geq\left(\begin{array}{c}4 \\ p-1\end{array}\right)$ for $p \geq 1$. In fact, one can derive a better bound using Theorem 1.1 as follows.

Set $W=\left(x_{1}^{2}, x_{1} x_{2}, x_{1} x_{3}^{3}, x_{1} x_{3}^{2} x_{4}, x_{1} x_{3}^{2} x_{5}\right)$. The Betti diagram of $S / W$ is the following:

$$
\begin{array}{ccccccc}
\text { total: } & 1 & 5 & 10 & 10 & 5 & 1 \\
0: & 1 & - & - & - & - & - \\
1: & - & 2 & 1 & - & - & - \\
2: & - & - & - & - & - & - \\
3: & - & 3 & 9 & 10 & 5 & 1
\end{array}
$$

No consecutive cancellations are possible. So by Theorem 1.1 it follows that for every homogeneous ideal $J$ with the same Hilbert function as $L$ we have that $\beta_{p}(S / J) \geq\left(\begin{array}{l}5 \\ p\end{array}\right)$ for $p \geq 0$

Example 1.7. Consider the ideal $I=\left(x_{n}^{a}, P\right)$, such that

(1) $P$ is a set of homogeneous polynomials;

(2) $x_{n}^{a-2+q}$ is a basis for the space $(S / P)_{a-2+q}$ for $q=0,1$.

We have that $\beta_{p}(S / I) \geq\left(\begin{array}{c}n-1 \\ p-1\end{array}\right)$ for $p \geq 1$.

Proof. We will show that Corollary 1.5 can be applied to the lexicographic ideal $L$ with the same Hilbert function as $I$. Since $x_{n}^{a-2}$ is a basis for $(S / P)_{a-2}$, it follows that it is a basis for $(S / L)_{a-2}$ as well. Similarly, $x_{n}^{a-1}$ is a basis for $(S / L)_{a-1}$. Therefore, $L$ has no minimal monomial generator in degree $a-1$. Furthermore, $x_{n}^{a}$ is a minimal monomial generator of $L$ in degree $a$. Clearly, $L$ has no minimal monomial generators in degree $a+1$. So Corollary 1.5 can be applied.

\section{ACKNOWLEDGMENTS}

Theorem 1.1 was proved in response to a question communicated to me by $\mathrm{C}$. Francisco. I would like to thank A. Geramita for his strong encouragement to write this paper. Further applications of Theorem 1.1 are provided by Geramita-HarimaMigliore-Shin [GHMS]; in particular, they have independently discovered and are using an argument such as the one in Proposition 1.2.

\section{REFERENCES}

[Ba] D. Bayer: The division algorithm and the Hilbert scheme, Ph.D. Thesis, Harvard University, 1982.

[Ei] D. Eisenbud: Commutative Algebra with a View Towards Algebraic Geometry, SpringerVerlag, New York, 1995. MR 97a:13001

[EK] S. Eliahou and M. Kervaire: Minimal resolutions of some monomial ideals, J. Algebra, 129 (1990), 1-25. MR 91b:13019

[ER] G. Evans and B. Richert: Possible resolutions for a given Hilbert function, Communications in Algebra 30 (2002), 897-906. MR 2002k:13024 
[GHMS] A. Geramita. T. Harima, J. Migliore, and Y. Shin: Some remarks on the Hilbert functions of level algebras, preprint.

[GHS1] A. Geramita. T. Harima, and Y. Shin: An alternative to the Hilbert function for the ideal of a finite set of points in $\mathbf{P}^{n}$, Illinois J. Math. 45 (2001), 1-23. MR 2002g:13004

[GHS2] A. Geramita. T. Harima, and Y. Shin: Decompositions of the Hilbert function of a set of points in $\mathbf{P}^{n}$, Canad. J. Math. 53 (2001), 923-943. MR 2002i:13019

[Ha] R. Hartshorne: Connectedness of the Hilbert scheme, Publications Mathématiques IHES 29 (1966), 5-48. MR 35:4232

[Ma] F. Macaulay: Some properties of enumeration in the theory of modular systems, Proc. London Math. Soc. 26 (1927), 531-555.

[Pa] K. Pardue: Deformation classes of graded modules and maximal Betti numbers, Illinois J. Math. 40 (1996), 564-585. MR 97g:13029

[Ri] B. Richert: Smallest graded Betti numbers, J. Algebra 244 (2001), 236-259. MR 2002g:13024

Department of Mathematics, Cornell University, Ithaca, New York 14853 